OPEN ACCESS

Edited by:

Petia D. Koprinkova-Hristova,

Institute of Information and Communication Technologies (BAS),

Bulgaria

Reviewed by: Jun $\mathrm{Ma}$

Lanzhou University of Technology,

China

Jiang Wang

Tianjin University, China

*Correspondence:

Raghottam M. Sattiger raghottam.ms@gmail.com

Received: 21 August 2019 Accepted: 21 February 2020 Published: 18 March 2020

Citation:

Sattigeri RM (2020) Action Potential: A Vortex Phenomena; Driving Membrane Oscillations. Front. Comput. Neurosci. 14:21. doi: 10.3389/fncom.2020.00021

\section{Action Potential: A Vortex Phenomena; Driving Membrane Oscillations}

\author{
Raghottam M. Sattigeri* \\ Department of Physics, Faculty of Science, The Maharaja Sayajirao University of Baroda, Vadodara, India
}

Hodgkin-Huxley $(\mathrm{HH})$ model has been one of the most successful electrical interpretation of nerve membrane which led to revolutions in the field of computational neuroscience. On the contrary, experimental observations indicate that, an Action Potential (AP) is accompanied with certain physiological changes in the nerve membrane such as, production and absorption of heat; variation of axon diameter, pressure and length. Although, in the early 1900's a Pressure Wave Theory was proposed by E. Wilke, but, due to lack of sophisticated experimental techniques it was left uncharted. Until recently, when Heimburg-Jackson, Hady-Machta and Rvachev, independently proposed Soliton Theory (thermodynamic interpretation of nerve membrane), Mechanical Surface Waves theory (electro-mechanical interpretation) and Rvachev Model (mechano-electrical activation of voltage gated sodium ion channels) respectively; encouraging a deviation from the traditional $\mathrm{HH}$ interpretation with justification for the physical changes in the nerve membrane observed experimentally. But, these theories lead to a "hit and miss" scenario because, they do explain certain features (increase/decrease in axon diameter) but miss to explain, correlation between the strength of stimuli and spike rate of AP. Bio-physical models of nerve membrane are thus important for enhancing our understanding regarding the governing dynamics of neural activities encompassing the experimental observations. A novel theory is proposed here which, unravels vortex ring formation due to ion currents in the intracellular and extracellular region leading to variation of pressure causing the increment/decrement in axon diameter. These formations manifest as membrane oscillations which are used to establish a correlation between the strength of stimuli and spike rate of AP. The theory proposed in this paper, brings a paradigm shift in our understanding of neural dynamics from a thorough bio-physical and physiological perspective with promising applications.

Keywords: action potential, nerve membrane, neurophysics, vortex theory, membrane oscillator theory, biophysical model

\section{INTRODUCTION}

In an attempt to understand the governing dynamics of neural activity, several models have been designed (Hodgkin and Huxley, 1952b; Harmon and Lewis, 1966; Debanne, 2004; Heimburg and Jackson, 2005, 2007; Bean, 2007; Kim et al., 2007; Andersen et al., 2009; Rvachev, 2010; Fields, 2011; Tyler, 2012; Debanne et al., 2013; Mueller and Tyler, 2014; Shrivastava, 2014; El Hady and Machta, 2015; Engelbrecht et al., 2016). These models are based on different interpretations of 
Action Potential (AP) (Figure 1A) which govern the manifestation of microscopic functions of a neuron on a macroscopic scale in the brain. Hodgkin and Huxley $(\mathrm{HH})$ (Hodgkin and Katz, 1949; Hodgkin and Huxley, 1952a,b; Hodgkin, 1964a,b) established first model which interprets $\mathrm{AP}$ as a purely electric phenomena. Although this model led to revolutions in the field of computational neuroscience, it could not successfully incorporate other physiological features observed experimentally which accompanied AP in a neuron (Hill, 1950; Abbott et al., 1958; Julian and Goldman, 1962; Biondi et al., 1972; Hill et al., 1977; Iwasa and Tasaki, 1980; Iwasa et al., 1980; Tasaki and Iwasa, 1982; Terakawa, 1985; Tasaki et al., 1989; Tasaki and Byrne, 1992a,b; Galbraith et al., 1993; Tasaki, 1999). Decades after the seminal $\mathrm{HH}$ interpretation, Soliton Theory (ST) by Heimburg-Jackson (Heimburg and Jackson, 2005, 2007), Mechanical Surface Wave (MSW) by Hady-Machta (El Hady and Machta, 2015) and the Mechano-Electrical activation of sodium channels in Rvachev Model (Rvachev, 2010) addressed AP from a physiological perspective which incorporated the empirical observations. Although these theories explain the phenomena of
AP to a great accuracy but, not in entirety. New approximations are thus necessary for a thorough interpretation of AP which will facilitate the development of more accurate neuronal models and further our understanding about the nature of nerve membrane which lies at the foundation of various neuronal activities resulting in a complex brain function.

The model proposed by Heimburg-Jackson, suggests that, AP exhibits a solitonic behavior i.e., AP exerts a pressure perpendicular to the surface of nerve membrane and travels down the axon without any dissipation (Heimburg and Jackson, 2005, 2007). Also, this model treats generation of heat in the nerve membrane associated with the event of AP in a thermodynamic regime. Overall, this model does explain and indicate toward an increment in the axon diameter which is accompanied by the absorption and emission of heat by the nerve membrane but, it does not explain the decrement in axon diameter which follows subsequently. The thermodynamic and solitonic interpretation is quite useful for the investigation of collisions between two AP in a nerve membrane (Budvytyte et al., 2014).

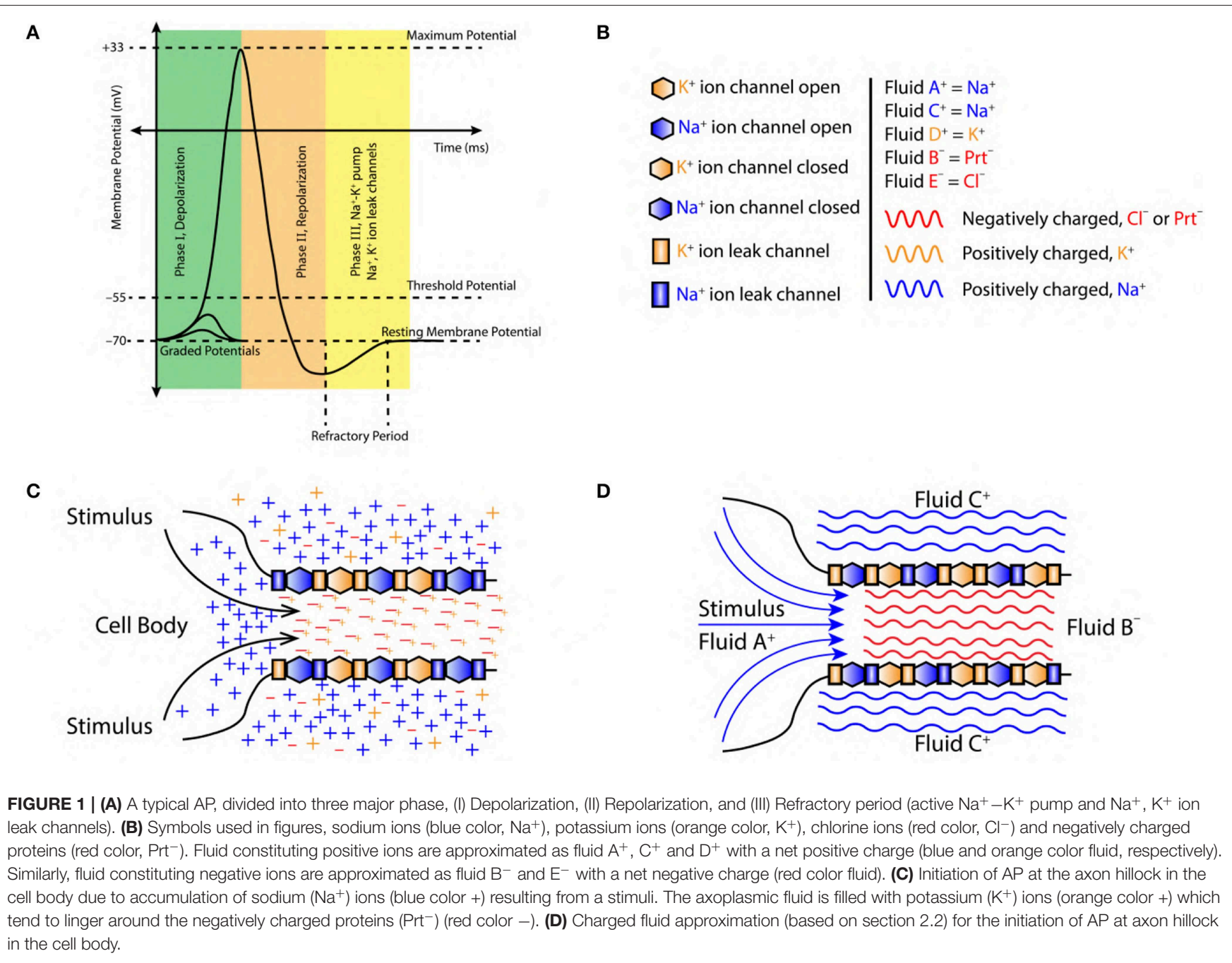


The electro-mechanical model proposed by Hady-Machta suggests that, AP is driven by electric constriction of a dielectric axon which indicates electro-mechanical origin of AP (confirmed computationally) (El Hady and Machta, 2015). This model successfully explains the origin of mechanical and thermal variations in the nerve membrane but, does not explicitly relate, the contribution of ion currents to the resulting mechanical changes in the nerve membrane. On the other hand, a rather versatile model proposed by Rvachev (2010), treats AP as a phenomena driven by the mechano-electrical activation of voltage gated sodium channels. This model successfully addresses physiological changes in nerve membrane (such as variation in mechanical, optical and thermal properties) to some extent the role of ion currents in the event of an AP but does not correlate, the spike rate (frequency) of AP with the strength of stimuli.

The models proposed so far result from, purely electric/ mechanical/thermodynamic/electro-mechanical/mechano-

electrical interpretation of the nerve membrane during the event of an AP. This has led to a broad bifurcation of theoretical models into two regimes, (i) electrical and (ii) non-electrical. In this paper, a new interpretation of nerve membrane pertaining to the event of AP is proposed which brings a paradigm shift in our understanding of nerve physiology and the underlying dynamics. Retaining the thermodynamic/electro-mechanical/mechanoelectrical treatment of nerve membrane (Heimburg and Jackson, 2005, 2007; Rvachev, 2010; El Hady and Machta, 2015) to address the absorption and emission of heat during the transport of AP, we focus on the role of ion currents across the nerve membrane during the event of an AP. The resulting interpretation unravels a vortex phenomena governing the formation and propagation of an AP through the axon due to peristaltic motion of the nerve membrane. Mechanical changes in the axon (such as variation of axon pressure, diameter and length) due to stress and strain result from, the pressures built-up in the intracellular and extracellular region due to vortex ring formation during depolarization (in the intracellular region) and repolarization (in the extracellular region) states, respectively. The fascinating outcome of this theory is that, the vortex ring formation drives nerve membrane into oscillations about its equilibrium position. This prediction enables us to establish a correlation between the strength of stimuli and spike rate (frequency) of AP, which has not been addressed thoroughly so far. Also, the proposed model is more realistic in terms of its broad umbrella which encompasses across every important facet of the nerve physiology pertaining to AP.

\section{VORTEX THEORY (VT)}

\subsection{Hypothesis}

Ion currents across the nerve membrane during, depolarization and repolarization phase of an AP form vortex ring.

\subsection{Assumptions}

Certain approximations and schematics will be followed to establish the VT for AP. These approximations neglect the complexities of molecular cell biology and are rather addressed from a macroscopic bio-physical perspective. This will serve as a prelude to the VT.
Elementary physics suggests that, a charge in motion gives rise to current (Halliday et al., 1995); ion currents across the membrane during, generation and propagation of AP are one such example in biological systems. These currents have not been addressed explicitly through the, ST, and MSW models discussed previously (section 1). It is necessary to incorporate this (ion currents) key feature of a nerve membrane to completely justify the phenomena of AP from a physiological perspective. For this purpose, certain approximations are made as follows:

1. The intracellular and extracellular regions of a neuron are made up of incompressible fluids (Figure 1D).

2. These fluids are composed of charged ions (sodium $\mathrm{Na}^{+}$, potassium $\mathrm{K}^{+}$, chlorine $\mathrm{Cl}^{-}$) and proteins $\mathrm{Prt}^{-}$ (Figures 1B,C). The net charge of the fluid is governed by the concentration of ions (Figure 2B). This implies, positive/negative fluids are dominated by the positive/negative ions, respectively (Figure 1C).

3. The ion currents across the nerve membrane are approximated as, flow of charged fluid through the nerve membrane.

4. The forces governing fluid flow across the nerve membrane are, diffusive and coulombic in nature.

5. Interactions with charged entities (other than those mentioned here) in the extracellular and intracellular region are neglected.

6. The nerve structure is unipolar with the shape of axon postaxon hillock following cylindrical symmetry.

\subsection{Ion Schematics}

\subsubsection{Pre-depolarization}

The concentration of $\mathrm{Na}^{+}, \mathrm{K}^{+}$, and $\mathrm{Cl}^{-}$ions in the extracellular region before depolarization are, $\mathrm{C}_{\mathrm{Na}^{+}}, \mathrm{C}_{\mathrm{K}^{+}}$, and $\mathrm{C}_{\mathrm{Cl}^{-}}$, respectively. These concentrations are related as, $\mathrm{C}_{\mathrm{Na}^{+}}>\mathrm{C}_{\mathrm{Cl}^{-}}$ $>\mathrm{C}_{\mathrm{K}^{+}}$(Figure 2B). Similarly, the concentration of $\mathrm{K}^{+}, \mathrm{Na}^{+}$ ions and proteins with net negative charge $\left(\mathrm{Prt}^{-}\right)$in the intracellular region are, $\mathrm{C}_{\mathrm{K}^{+}}, \mathrm{C}_{\mathrm{Na}^{+}}$, and $\mathrm{C}_{\mathrm{Prt}^{-}}$, respectively. They are related as, $\mathrm{C}_{\mathrm{Na}^{+}}<\mathrm{C}_{\mathrm{K}^{+}}<\mathrm{C}_{\mathrm{Prt}^{-}}$(Figure 2B). Thus, in the pre-depolarization state, the extracellular region is electropositive and the intracellular region is electro-negative governed by the concentration gradient (as shown in Figure 2A). This is represented by the net charge state, in the extracellular region (by the virtue of $\mathrm{Na}^{+}$ions) and in the intracellular region (by the virtue of $\mathrm{Prt}^{-}$) as, $\left.\mathrm{Q}_{\text {extra }}{ }\right|_{\mathrm{Na}}+$ and $\left.\mathrm{Q}_{\text {intra }}{ }^{-}\right|_{\text {Prt }^{-}}$, respectively (Figure $\mathbf{2 B}$ ).

\subsubsection{Phase I, Depolarization}

During depolarization (Figure 1A), due to diffusion of $\mathrm{Na}^{+}$ ions into the intracellular region (Lodish et al., 1995; Purves et al., 2008; Hall, 2010), the ion concentrations in extracellular and intracellular region change to, $\mathrm{C}_{\mathrm{K}^{+}}<\mathrm{C}_{\mathrm{Na}^{+}}<\mathrm{C}_{\mathrm{Cl}^{-}}$and $\mathrm{C}_{\mathrm{Na}^{+}}>\mathrm{C}_{\mathrm{K}^{+}}>\mathrm{C}_{\mathrm{Prt}^{-}}$, respectively (Figure $2 \mathrm{~B}$ ). This makes the intracellular region electro-positive and the extracellular region electro-negative due to the reversal of concentration gradient (as shown in Figure 2A). The net charge state in the extracellular region (by the virtue of $\mathrm{Cl}^{-}$ions) and in the intracellular region 


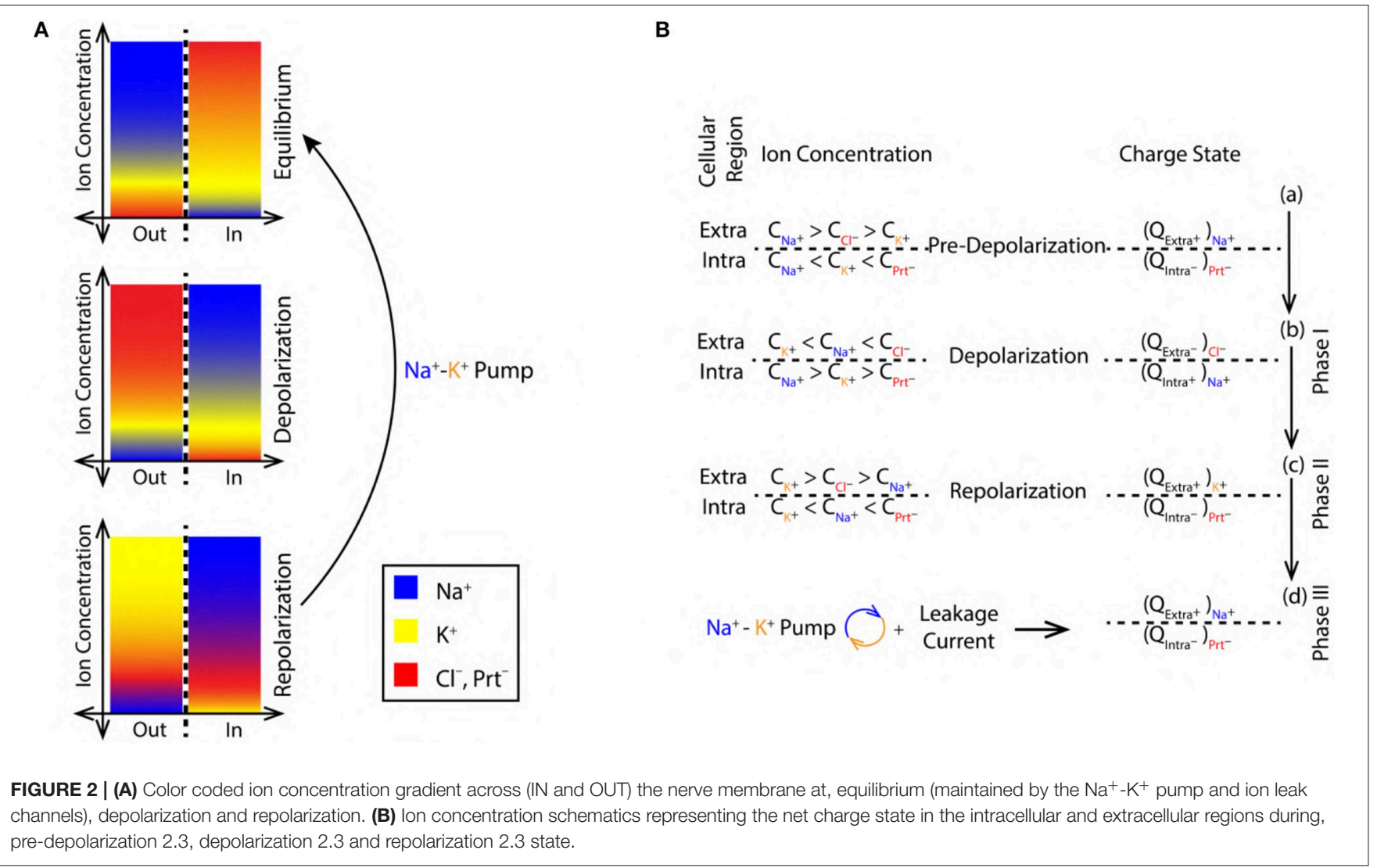

(by the virtue of $\mathrm{Na}^{+}$ions) is represented as, $\mathrm{Q}_{\mathrm{extra}}{ }^{-} \mathrm{Cl}^{-}$and $\mathrm{Q}_{\text {intra }}+\left.\right|_{\mathrm{Na}}+$ (Figure 2B).

\subsubsection{Phase II, Repolarization}

During repolarization (Figure 1A), due to the diffusion of $\mathrm{K}^{+}$ ions into the extracellular region (Lodish et al., 1995; Purves et al., 2008; Hall, 2010) leaving behind the negatively charged $\mathrm{Prt}^{-}$, the ion concentrations in the extracellular and intracellular region change to, $\mathrm{C}_{\mathrm{K}^{+}}>\mathrm{C}_{\mathrm{Cl}^{-}}>\mathrm{C}_{\mathrm{Na}^{+}}$and $\mathrm{C}_{\mathrm{K}^{+}}<\mathrm{C}_{\mathrm{Na}^{+}}<\mathrm{C}_{\mathrm{Prt}^{-}}$, respectively (Figure $2 \mathrm{~B}$ ). This makes the intracellular region electro-negative and the extracellular region electro-positive due to concentration gradient (as shown in Figure 2A). The net charge state in the extracellular region (by the virtue of $\mathrm{K}^{+}$ ions) and in the intracellular region (by the virtue of $\mathrm{Prt}^{-}$) is represented as, $\mathrm{Q}_{\text {extra }}+\left.\right|_{\mathrm{K}^{+}}$and $\left.\mathrm{Q}_{\text {intra }}{ }^{-}\right|_{\mathrm{Prt}^{-}}$, respectively (Figure 2B).

\subsubsection{Phase III, Restoration of Pre-depolarization State}

Figure 2B represents the refractory period (RF) (Figure 1A) when the Pre-Depolarization state is restored by the $\mathrm{Na}^{+}$. $\mathrm{K}^{+}$Pump and the $\mathrm{Na}^{+}, \mathrm{K}^{+}$ion leak channels (Lodish et al., 1995; Purves et al., 2008; Hall, 2010). Represented by the net charge state as, $\mathrm{Q}_{\text {extra }}+\left.\right|_{\mathrm{Na}}+$ and $\left.\mathrm{Q}_{\text {intra }^{-}}\right|_{\mathrm{Prt}^{-}}$(Figure 2B). This restores and maintains the electro-chemical gradient ready for next stimulus.

\subsection{Pressure Schematics}

The ions are in a state of constant motion/jiggle at room temperature by the virtue of thermal energy (Halliday et al., 1995) in the intracellular and extracellular region. In such state, they can freely interact with any other components (such as proteins, other ions etc.) in the intracellular as well as the extracellular region. This drives the system toward a state of dynamic equilibrium due to a balance in the intracellular and extracellular pressure. This state of the system gets perturbed during the event of an AP. At equilibrium (resting state), pressure is balanced on either sides of the membrane i.e., ${ }^{e q} P_{\text {out }}={ }^{e q} P_{\text {in }}$ (Figure 3A). In the extracellular region, the pressure is composed of partial pressures (PP) due to $\mathrm{Na}^{+}, \mathrm{Cl}^{-}$and $\mathrm{K}^{+}$ions (Equation 1). Similarly, the intracellular region is composed of $\mathrm{PP}$ due to $\mathrm{Prt}^{-}, \mathrm{K}^{+}$, and $\mathrm{Na}^{+}$ions (Equation 2). The sequence of pressure variation is in concurrence with the ion concentration schematics discussed in previous section 2.3. An AP drives the system away from its dynamic equilibrium state under the influence of the diffusive forces and the ion concentration gradients (Figure 2A) across the membrane (discussed in section 2.3). Then, during depolarization, due to influx of $\mathrm{Na}^{+}$ions in the intracellular region, $\mathrm{PP}$ in extracellular region decreases (Equation 3) and increases proportionally in the intracellular region (Equation 4). Where, $\delta$ represents a finite change in the PP. Similarly, during repolarization, due to the flow of $\mathrm{K}^{+}$ions into the extracellular region, the PP in intracellular region reduces (Equation 6) and increases PP proportionally in the extracellular 
A

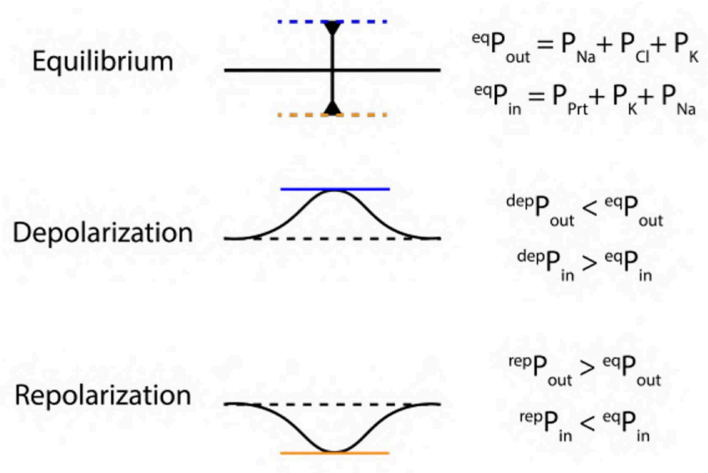

B

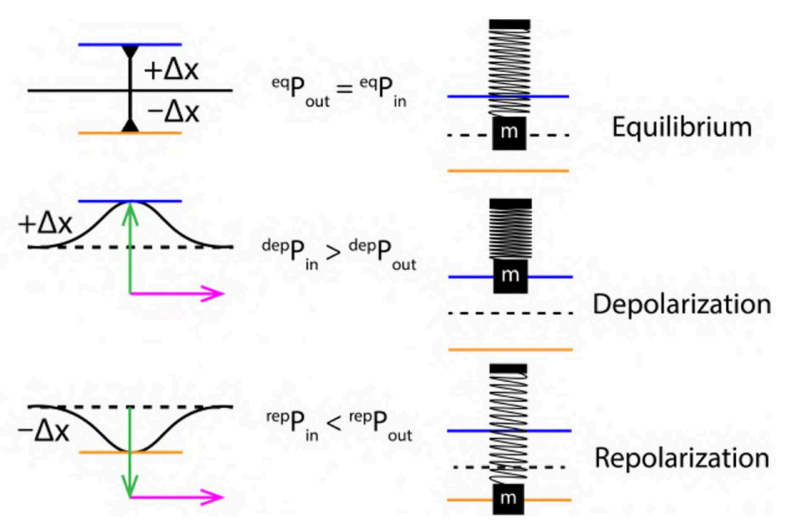

FIGURE 3 | (A) Schematics of extracellular $\left(\mathrm{P}_{\text {out }}\right)$ and intracellular $\left(\mathrm{P}_{\text {in }}\right)$ pressure during, equilibrium (eqP), depolarization ( $\left.{ }^{\text {dep }} \mathrm{P}\right)$ and repolarization ( ${ }^{\text {rep } P)}$ states. (B) Schematic SM approximation of a small section of nerve membrane during, equilibrium, depolarization and repolarization. Green arrow indicates the force exerted due to variation in PP discussed in section 2.4 and the magenta arrow indicates the direction of propagation of the AP.

region (Equation 5).

$$
\begin{aligned}
{ }^{e} \mathrm{e}_{\mathrm{P}_{\text {out }}} & =\mathrm{P}_{\mathrm{Na}}+\mathrm{P}_{\mathrm{Cl}}+\mathrm{P}_{\mathrm{K}} \\
{ }^{e} \mathrm{P}_{\text {in }} & =\mathrm{P}_{\mathrm{Prt}}+\mathrm{P}_{\mathrm{K}}+\mathrm{P}_{\mathrm{Na}} \\
{ }^{d e p} \mathrm{P}_{\text {out }} & ={ }^{e} \mathrm{P}_{\text {out }}-\delta \mathrm{P}_{\mathrm{Na}} \\
{ }^{d e p} \mathrm{P}_{\text {in }} & ={ }^{e} \mathrm{P}_{\text {in }}+\delta \mathrm{P}_{\mathrm{Na}} \\
{ }^{\text {rep }} \mathrm{P}_{\text {out }} & ={ }^{d e} \mathrm{P}_{\text {out }}+\delta \mathrm{P}_{\mathrm{K}} \\
{ }^{\text {rep }} \mathrm{P}_{\text {in }} & ={ }^{d e} \mathrm{P}_{\text {in }}-\delta \mathrm{P}_{\mathrm{K}}
\end{aligned}
$$

These changes in PP exert a force perpendicular to the nerve membrane displacing it from its equilibrium position outward (during depolarization) and inward (during repolarization) (Figure 3A). Such variation in pressure has been discussed previously in experimental studies with plant cells (Chara braunii internodes) (Fillafer et al., 2018), this implies that, the justification for variation in pressure provided above is very much plausible.

\subsection{Fluid Schematics}

With the thorough background of sections 2.2, 2.3, and 2.4, the increase and decrease in the diameter of nerve membrane accompanying AP are shown in Figures 4A,B due to coulomb interactions among the positively charged fluids $\mathrm{A}^{+}, \mathrm{C}^{+}$, $\mathrm{D}^{+}$(section 2.2) and negatively charged fluids $\mathrm{B}^{-}$and $\mathrm{E}^{-}$ (section 2.2) during the event of AP discussed in the following section 2.6.

\subsection{Vortex Formation}

Vortex formation will be discussed in the background of theoretical approximations and schematics disscussed in sections 2.2, 2.3, and 2.4. In the following sections, a qualitative analysis of the hypothesis (section 2.1) is established which indicates that, the cascade of events pertaining to formation and propagation of AP create a vortex ring of ionic fluid. Vortex ring formation is a physical phenomena generally observed in fluids (liquids/gases) (Shariff and Leonard, 1992). It has been extensively studied from a biological perspective to understand the dynamics of mitral valve and left ventricle of heart (Bellhouse and Bellhouse, 1969; Bellhouse, 1972; Reul et al., 1981; Pasipoularides et al., 1984; Seo et al., 2014). These studies are based on the analysis of fluid dynamics in terms of the velocity vector of the fluid particles. Contrary to this, VT proposed here; is based on the fluid like dynamics exhibited by the charged fluids (section 2.2), governed by the coulomb interactions and diffusive forces. This helps to incorporate the ion currents across the membrane which are intricately involved in the initiation and propagation of AP.

\subsubsection{Stimuli}

The excitatory stimulus (inhibitory stimuli is neglected since it does not lead to an AP) increases the concentration of positive ions $\mathrm{Na}^{+}$in cell body (Lodish et al., 1995; Purves et al., 2008; Hall, 2010). As a result of this, the positive ions start accumulating at the axon hillock as shown in Figure 1C. In accordance with the "all or none law" (Adrian, 1914), at the axon hillock graded potentials (Figure 1A) are generated due to the influx of $\mathrm{Na}^{+}$ ions. When these graded potentials cross the threshold potential $(-55 \mathrm{mV})$, the $\mathrm{Na}^{+}$ion channels in the close proximity of axon hillock, undergo a conformational change $\left(\mathrm{Na}^{+}\right.$ion channels open/become active) (Catterall, 1995; Hille, 2001; Catterall et al., 2012). This triggers an AP which manifests itself as a vortex ring of charged fluid retaining the ring like structure as it travels through the axon (as shown in Figure 4C).

\subsubsection{Depolarization}

During depolarization (Figure 4C), the positively charged fluid $\mathrm{A}^{+}$(made of $\mathrm{Na}^{+}$ions in the intracellular region) is accelerated toward the axon terminal under the influence of coulomb attraction with negatively charged fluid $\mathrm{B}^{-}$(made of $\mathrm{Prt}^{-}$ proteins). Due to the flow of fluid $\mathrm{A}^{+}$into the intracellular region beyond the axon hillock, the voltage gated $\mathrm{Na}^{+}$ion channels get activated (Catterall, 1995; Hille, 2001; Catterall et al., 2012) leading to rapid influx of positively charged fluid $\mathrm{C}^{+}$(made of $\mathrm{Na}^{+}$ions in the extracellular region) driven by diffusive forces governed by the concentration gradient discussed in section 2.3. This event forms along a tri-junction surface of interaction 


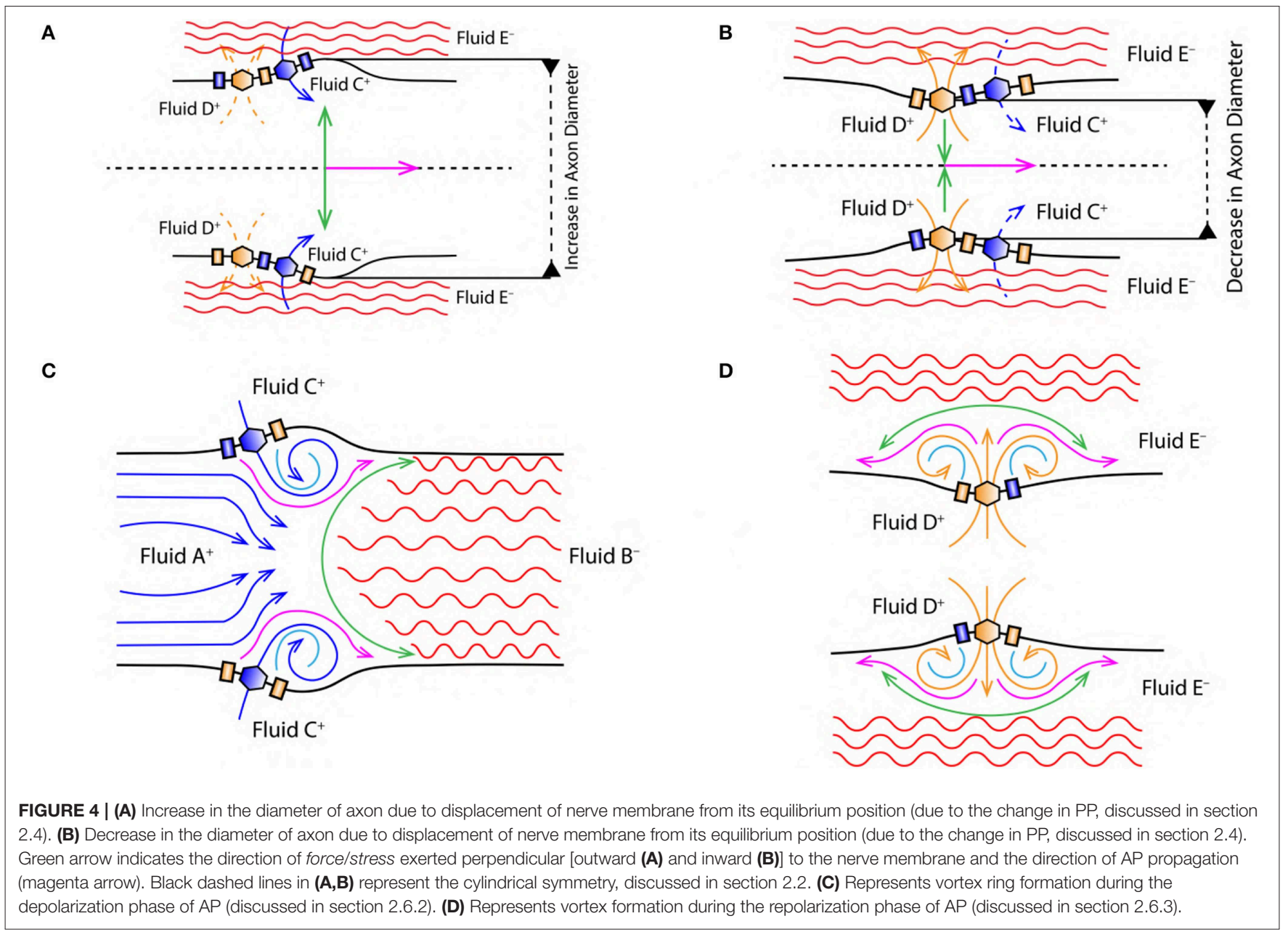

represented by the magenta line in Figure 4C. At a given instance of time in the depolarization phase of $\mathrm{AP}$, these interactions occur between (i) fluid $\mathrm{A}^{+}-\mathrm{C}^{+}$, (ii) fluid $\mathrm{A}^{+}-\mathrm{B}^{-}$and (iii) fluid $\mathrm{C}^{+}-\mathrm{B}^{-}$. Both, fluid $\mathrm{A}^{+}$and $\mathrm{C}^{+}$are accelerated toward the axon terminal due to the coulomb attraction with fluid $\mathrm{B}^{-}$ represented by green surface in Figure 4C. Also, fluid $\mathrm{A}^{+}$and $\mathrm{C}^{+}$interact under coulomb repulsion; pushing fluid $\mathrm{C}^{+}$toward the cell membrane (Figure 4C). Since fluid $\mathrm{C}^{+}$is pushed toward the membrane, it interacts under coulomb repulsion with the diffusing fluid $\mathrm{C}^{+}$along the sky-blue line as shown in Figure 4C. This forms a ring like structure (as shown in Figure 4C) which traverses down the axon retaining its shape. The ring thus formed is a vortex of fluid $\mathrm{C}^{+}\left(\mathrm{Na}^{+}\right.$ions). Hence, the motion of $\mathrm{AP}$ is a net effect of repulsion between fluid $\mathrm{A}^{+}-\mathrm{C}^{+}$and attraction of fluid $\mathrm{A}^{+}-\mathrm{C}^{+}$with fluid $\mathrm{B}^{-}$. This creates a extracellular region dominated by negatively charged fluid $\mathrm{E}^{-}$(made of $\mathrm{Cl}^{-}$ ions) and the intracellular region dominated by fluid $\mathrm{A}^{+}$and $\mathrm{C}^{+}$with net positive charge, depolarizing (as shown by reversal of ion concentration across the membrane in Figure 2A) the nerve membrane. The influx of fluid $\mathrm{C}^{+}$continues (expanding the axon as shown in Figure 4A) until the membrane reaches a maximum potential $(+33 \mathrm{mV})$ which, causes inactivation of the voltage gated $\mathrm{Na}^{+}$ion channels and activation of the voltage gated $\mathrm{K}^{+}$ion channels (Catterall, 1995; Hille, 2001; Catterall et al., 2012).

\subsubsection{Repolarization}

Activation of $\mathrm{K}^{+}$ion channels leads to diffusion of fluid $\mathrm{D}^{+}$(made of $\mathrm{K}^{+}$ions in the intracellular region) across the membrane in the extracellular region driven by concentration gradient (as shown in Figure 2B). The extracellular region is dominated by fluid $\mathrm{E}^{-}$due to depolarization (section 2.6.2). Hence, diffusion of fluid $\mathrm{D}^{+}$into the extracellular region is not only driven by the concentration gradient (Figure 2A) but also due to the coulomb attraction with fluid $\mathrm{E}^{-}$along the green line (Figure 4D). The velocity of fluid $\mathrm{D}^{+}$reduces (due to coulomb repulsion with a lower concentrations of fluid $\mathrm{C}^{+}$along the green line as in Figure 4D) as it travels farther from the nerve membrane and the successive diffusion of fluid $\mathrm{D}^{+}$spreads in outward direction along the magenta line governed by coulomb repulsion with fluid $\mathrm{D}^{+}$along the sky-blue line (Figure 4D). The fluid interactions during repolarization phase are hence summarized as interaction between, (i) fluid $\mathrm{D}^{+}-\mathrm{E}^{-}$, (ii) fluid $\mathrm{D}^{+}-\mathrm{D}^{+}$and (iii) slightly due to fluid $\mathrm{D}^{+}-\mathrm{C}^{+}$. This results in compression of the axon as shown in Figure 4B. 


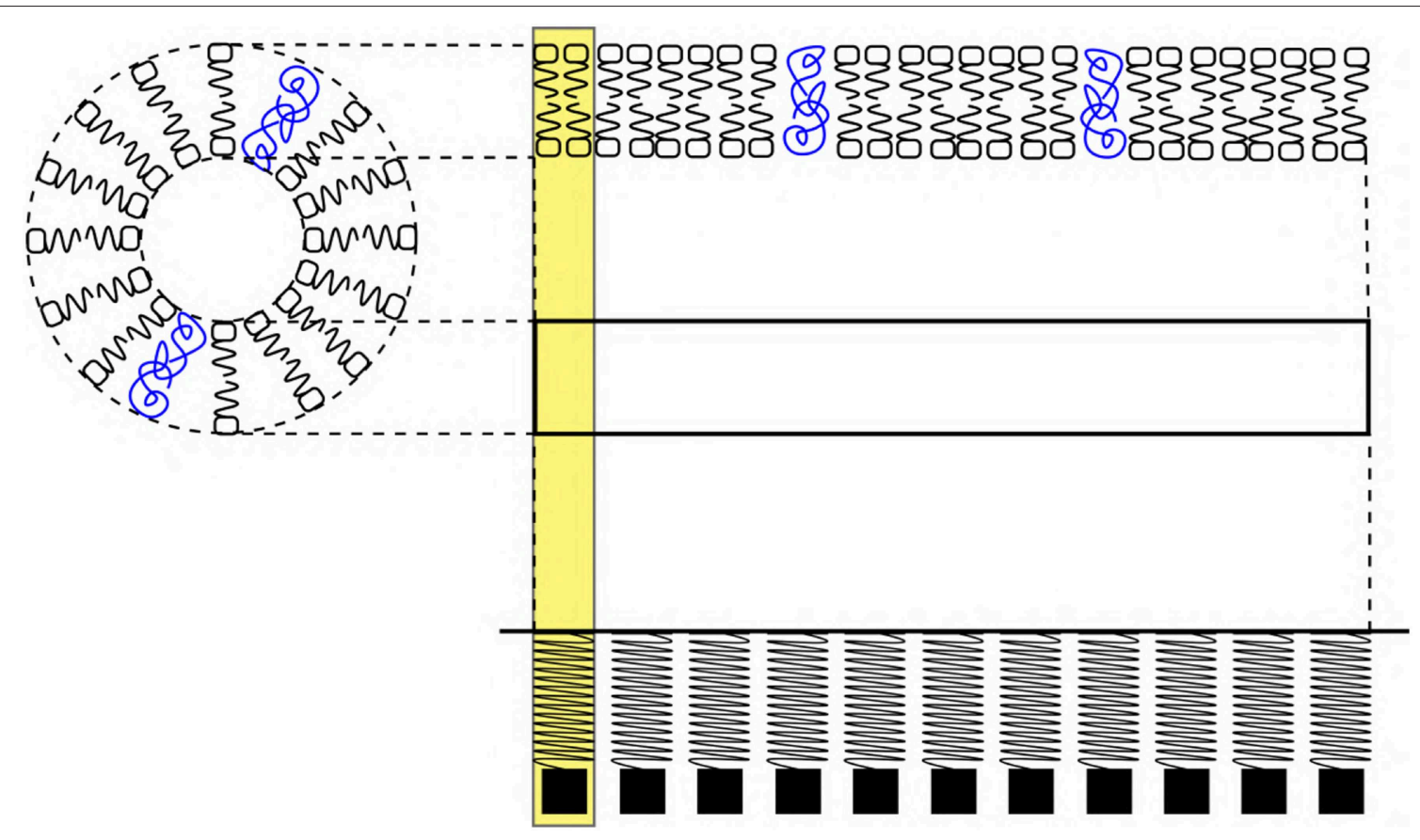

FIGURE 5 | (Left) Schematic cross sectional view of an axon. (Right) Phospholipid bilayer membrane schematic represents, a section (horizontal view) of the axon which is approximated as an elastic ribbon (white strip) which is similar to a linear arrangement of a SM system. The yellow highlight represents a unit length of the membrane approximated as a SM system. The blue colors scribbles represent ion channel proteins. The sections of ribbon are analogous to the SM system.

\section{MEMBRANE OSCILLATOR THEORY (MOT)}

Thorough qualitative analysis performed in section 2 unravels physical changes in the nerve membrane associated with the vortex ring formation in the intracellular and extracellular region. This section deals with, manifestation of physical changes in the nerve membrane as membrane oscillations.

\subsection{Hypothesis}

"Motion of AP is associated with membrane oscillations driven by, vortex ring formation in the intracellular and extracellular region."

\subsection{Assumptions}

1. The phospholipid bilayer membrane (Safran, 2018) (nerve membrane) is approximated as an elastic ribbon composed of "N" SM systems suspended from a rigid support (Figure 5) (the terms, membrane and ribbon will have synonymous meaning in the discussions hereof).

2. Vortex ring formation (section 2.6) exerts a force perpendicular to the ribbon (as discussed in section 2.4).

3. The displacements in ribbon due to such force is governed by the Hooke's Law (Hooke, 1635-1703).

4. These displacements occur within the elastic limit (i.e., stress $\propto$ strain) (Halliday et al., 1995).

5. These displacements are analogous to the simple harmonic motion of a typical Spring-Mass (SM) system (Figure 3B).
6. Elasticity (k) of the spring indicates elasticity of the ribbon which is ideally constant for a neuron (although it may vary due to diseases/disorders which are not considered here).

7. The mass (m) of the SM system (discussed in section 3.3) represents the net mass of a section of ribbon where the event of AP occurs (Figure 5). This mass is assumed to be constant.

8. A single cycle of oscillation performed by the SM system or ribbon corresponds to a single event of AP.

9. The spike rate (frequency) of $A P\left(f_{A P}\right)$ is equal to the frequency of ribbon oscillations $\left(f_{\text {ribbon }}\right)$.

10. Strength of stimuli (E) is directly proportional to the concentration of ions ([C]) in the cell body.

\subsection{Schematics}

A SM system typically made up of, a mass (m) suspended with the help of a massless spring supported by a rigid surface is considered (as shown in Figure 6, 1) (Halliday et al., 1995). At equilibrium, the position of mass $(\mathrm{m})$ is represented by dashed black line (Figure 6, 1). When an external force (F) acts on such a mass, it gets displaced by a distance $+\Delta \mathrm{x}$ which is proportional to the applied force (F), Equation (7). Then, according to Hooke's Law (Hooke, 1635-1703), the force and displacement are related as in Equation (8) where, $k$ is a constant of proportionality which defines elasticity of the spring. The sign in Equation (8) is positive $(+)$ during depolarization (due to displacement $+\Delta \mathrm{x}$ ) and negative during repolarization (due to displacement $-\Delta \mathrm{x})$. These displacements are discussed in terms of the stress (perpendicular) and strain (transverse) (Figure 7) due to push 


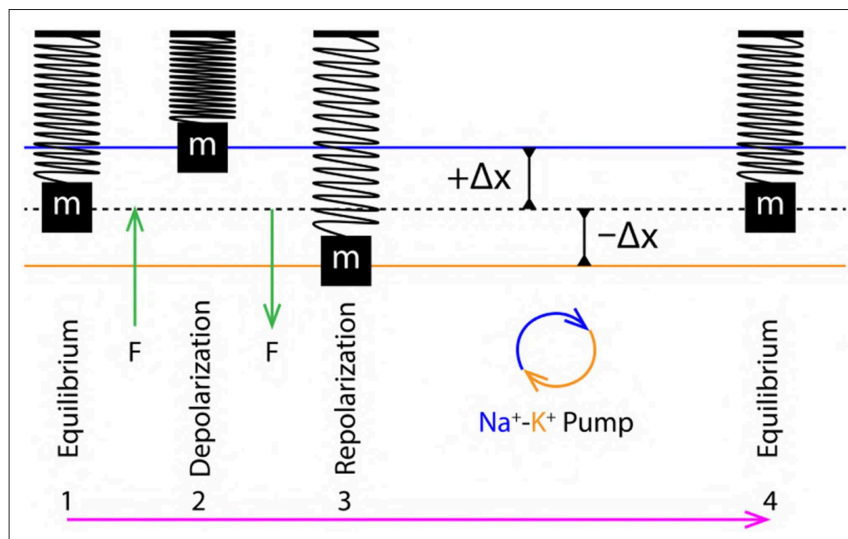

FIGURE 6 | 1 to 4 are the sequence of events representing a single cycle of membrane oscillation corresponding to a single AP.

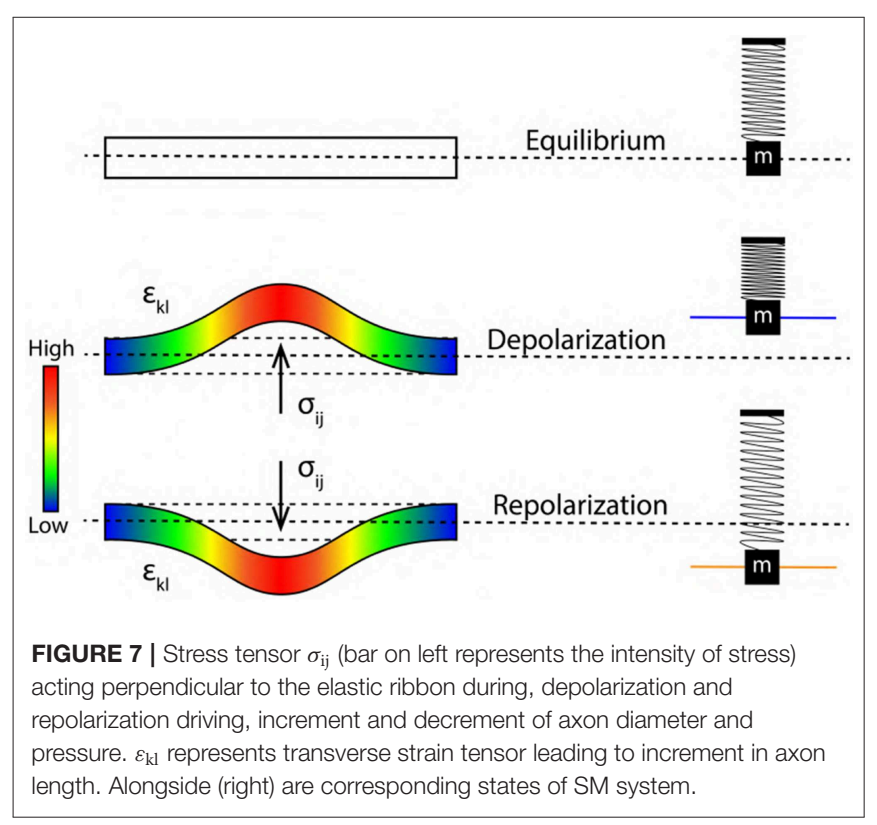

(/pull) and stretch in the ribbon, respectively (discussed in section 3.4).

$$
\begin{aligned}
& \mathrm{F} \propto \Delta \mathrm{x} \\
& \mathrm{F}= \pm \mathrm{k} \Delta \mathrm{x}
\end{aligned}
$$

From section 2.4, the variation of PP in the extracellular and intracellular region creates a to and fro motion of the nerve membrane (Figure 3A). This motion can be decomposed into three phases (Figure 6) of AP (Figure 1A), (i) Equilibrium, (ii) Depolarization and (iii) Repolarization. According to the variation in $\mathrm{PP}$, at equilibrium ${ }^{e q} \mathrm{P}_{\mathrm{out}}$ is equal to ${ }^{\mathrm{eq}} \mathrm{P}_{\mathrm{in}}$. This state changes rapidly during depolarization since, ${ }^{\text {dep }} \mathrm{P}_{\text {in }}$ $>{ }^{\text {dep }} \mathrm{P}_{\text {out }}$. The increment of pressure in the intracellular region exerts a force (F) perpendicular to the membrane which displaces it by $+\Delta \mathrm{x}$ about its equilibrium position in outward direction, this is represented by compression of the SM system (Figure 6, 2). Similarly, during repolarization, the pressure in extracellular region changes rapidly as ${ }^{\text {rep }} \mathrm{P}_{\text {in }}<$ ${ }^{r e p} \mathrm{P}_{\text {out }}$. The increment of pressure in the extracellular region exerts a force (F) perpendicular to the nerve membrane displacing it by $-\Delta \mathrm{x}$ about its equilibrium position but in opposite direction (inward) as compared to that during depolarization. This is represented by expansion of the SM system (Figure 6, 3). Since it is approximated (section 3.2) that, the membrane displacements lie within the elastic limits, it ensures that, the membrane does not rupture due to such rapid changes in pressure which occur on a time scale of milliseconds.

\subsection{Oscillations}

With reference to previous section 3.2, a quantitative analysis describing membrane oscillations is discussed below in terms of changes in the elastic ribbon (Figure 5). Also, the physical origin of spike rate (frequency) of AP is discussed, relating it to the frequency of ribbon oscillations $\left(f_{\text {ribbon }}\right)$. This unravels for the very first time, a mechanism by which we can interpret encoding and transmission of information in a neuron. Figure 6 represents a single cycle of membrane oscillation as discussed in section 3.3. Since, these oscillations are governed my the Hooke's Law, the restoring force acting on the system is analogous to the activation of $\mathrm{Na}^{+}-\mathrm{K}^{+}$pump which restores the membrane potential back to the resting state (Figure 1A). Thus, the time taken in transition from phase 3 to 4 represents the refractory period (Figure 6). This period depends on the elasticity of membrane defined by, the constant of proportionality in Equation (8) which is assumed to be invariant (section 3.2). Since, a ribbon (nerve membrane) is an example of continuous media (Sedov, 1997), the terms in Equation (8) are replaced by second order tensors for continuous media as in Equation (9). Figure 5 represents the elastic ribbon approximation of the phospholipid bilayered nerve membrane which is subjected to stress $\left(\sigma_{\mathrm{ij}}\right)$ analogous to the force $(\mathrm{F})$ in Equation (8), this leads to transverse strain $\left( \pm \varepsilon_{\mathrm{kl}}\right)$ in the ribbon analogous to displacement $( \pm \Delta x)$ in Equation (8). Similar to the constant of proportionality in Equation (8), $c_{\mathrm{ijkl}}(\mathrm{i}, \mathrm{j}=1,2,3)$ in Equation (9) is a fourth order stiffness/elasticity tensor. Figure 7 represents a elastic ribbon under application of a perpendicular stress, leading to transverse strain.

$$
\sigma_{\mathrm{ij}}= \pm \sum_{\mathrm{k}=1}^{3} \sum_{\mathrm{l}=1}^{3} \mathrm{c}_{\mathrm{ijkl}} \varepsilon_{\mathrm{kl}}
$$

Figure 8 represents a cross sectional view of the membrane oscillations with variation in axon radius $\left(r=r_{0}\right.$ at equilibrium) as $\mathrm{r}>\mathrm{r}_{0}$ during depolarization and $\mathrm{r}<\mathrm{r}_{0}$ during repolarization. A single event of AP (depolarization, $+\Delta \mathrm{x}$ and repolarization, $-\Delta \mathrm{x})$ are represented by the variation of axon diameter approximated as a ribbon with the corresponding SM states. Since the two, elastic ribbon and SM system are analogous, the frequency of ribbon oscillation $\mathrm{f}_{\text {ribbon }}$ represents the spike rate of AP (section 3.2). Based on the approximations of section 3.2, the elasticity $(\mathrm{k})$ and mass $(\mathrm{m})$ of a unit length of ribbon (Figure 5) are constant, this makes the frequency of oscillations dependent indirectly on $\mathrm{k}$ and $\mathrm{m}$. Due to this constrain we cannot establish a relation between the spike rate (frequency) of AP and strength of 

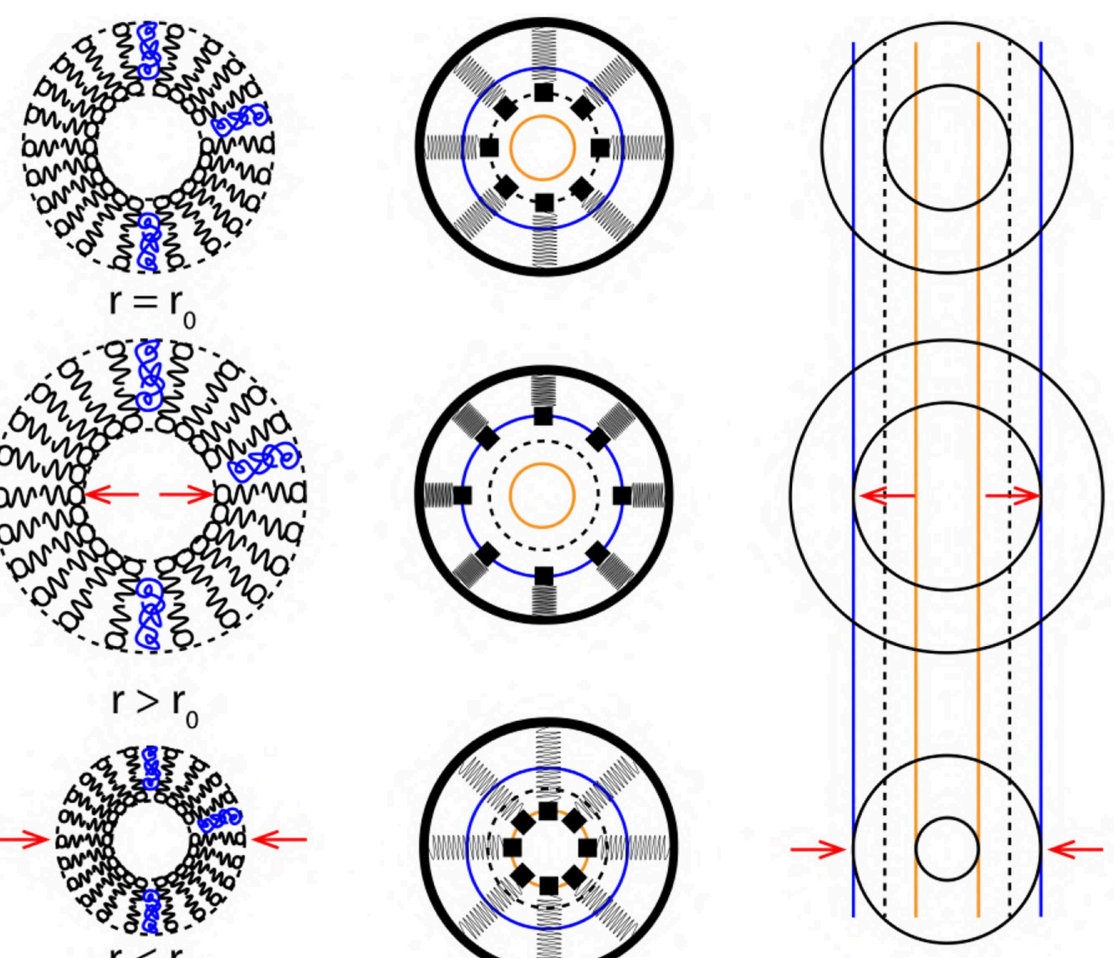

\section{(i) Equilibrium \\ $r=r_{0}$}
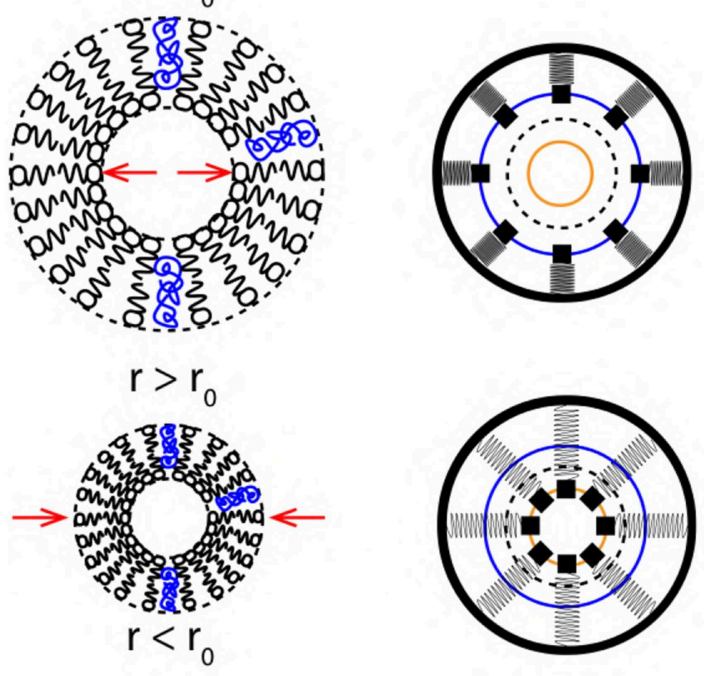

FIGURE 8 | Cross sectional view (top to bottom) of membrane oscillations during a single cycle of AP. (left to right) Membrane approximations (phospholipid structure $>\mathrm{SM}$ system > elastic ribbon), representing membrane oscillations in terms of variation of axon radius ( $r$ ) about its equilibrium position ( $\left.r_{0}\right)$.

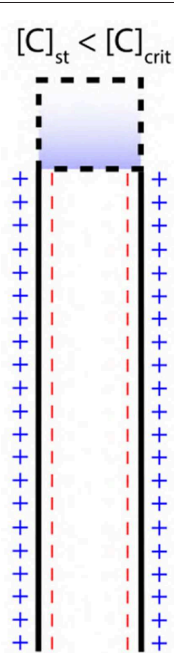

A

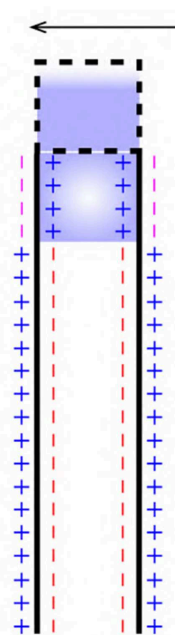

B

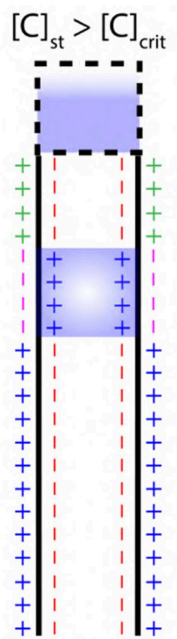

C

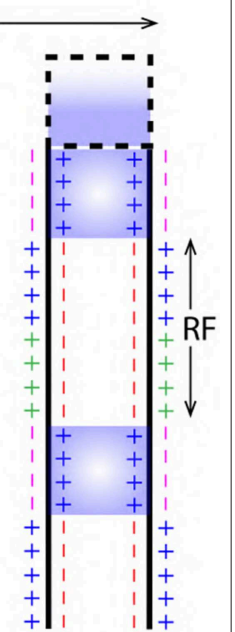

D
FIGURE 9 | (A) Concentration of ions due to stimuli $[\mathrm{C}]_{\text {st }}$ in the cell body is less than the critical concentration $[C]_{\text {crit }}$ required to generated an $\mathrm{AP}$. (B,C) AP initiation and propagation when, the $[C]_{s t}$ is greater than $[C]_{\text {crit }}$. (D) Two successive events of AP resolved by a refractory period (RF).

stimuli directly. This is solved in the following section, in order to establish a correlation between the strength of stimuli and spike rate of AP.

\subsection{Frequency}

The strength of stimuli (E) and the corresponding concentration of ions ([C]) in the cell body are related as in Equation (10) based on the assumptions of section 3.2. This implies that, if a neuron is stimulated for time $(t)$ then, the concentration of ions in the cell body increases due to the activation of ion channels (which can be, voltage, ligand and mechanically gated). A critical concentration $\left([\mathrm{C}]_{\text {crit }}\right.$ ) of ions in cell body corresponds to, the value of threshold potential (Figure 1A) and is necessary for the initiation of an AP (Figure 9). Then, the critical strength of the stimuli $\mathrm{E}_{\text {crit }}$ defines, a threshold strength of stimuli required for a successful initiation of AP.

$$
\mathrm{E} \propto[\mathrm{C}]
$$

During depolarization, the concentration of ions in cell body due to stimuli $[\mathrm{C}]_{\text {st }}$ exceeds $[\mathrm{C}]_{\text {crit }}$ (as in Figure 9B). Then, a relation between net concentration of ions due to stimuli ${ }^{\text {net }}[\mathrm{C}]_{\mathrm{st}}$ and $[\mathrm{C}]_{\text {crit }}$ can be established as in Equation (11) where, $\beta$ is a multiplication factor.

$$
{ }^{\text {net }}[\mathrm{C}]_{\mathrm{st}}=\beta[\mathrm{C}]_{\mathrm{crit}}
$$

Since it is known that, an AP is generated by recurrent attempts of graded potentials (Figure 1A) (Lodish et al., 1995; Hall, 2010), the net concentration ${ }^{\text {net }}[\mathrm{C}]_{\text {st }}$ due to the stimuli from neighboring neurons (A, B, C, D, E) (Figure 10) can be written as a sum of individual concentrations as in Equation (12) (Figure 10). This 


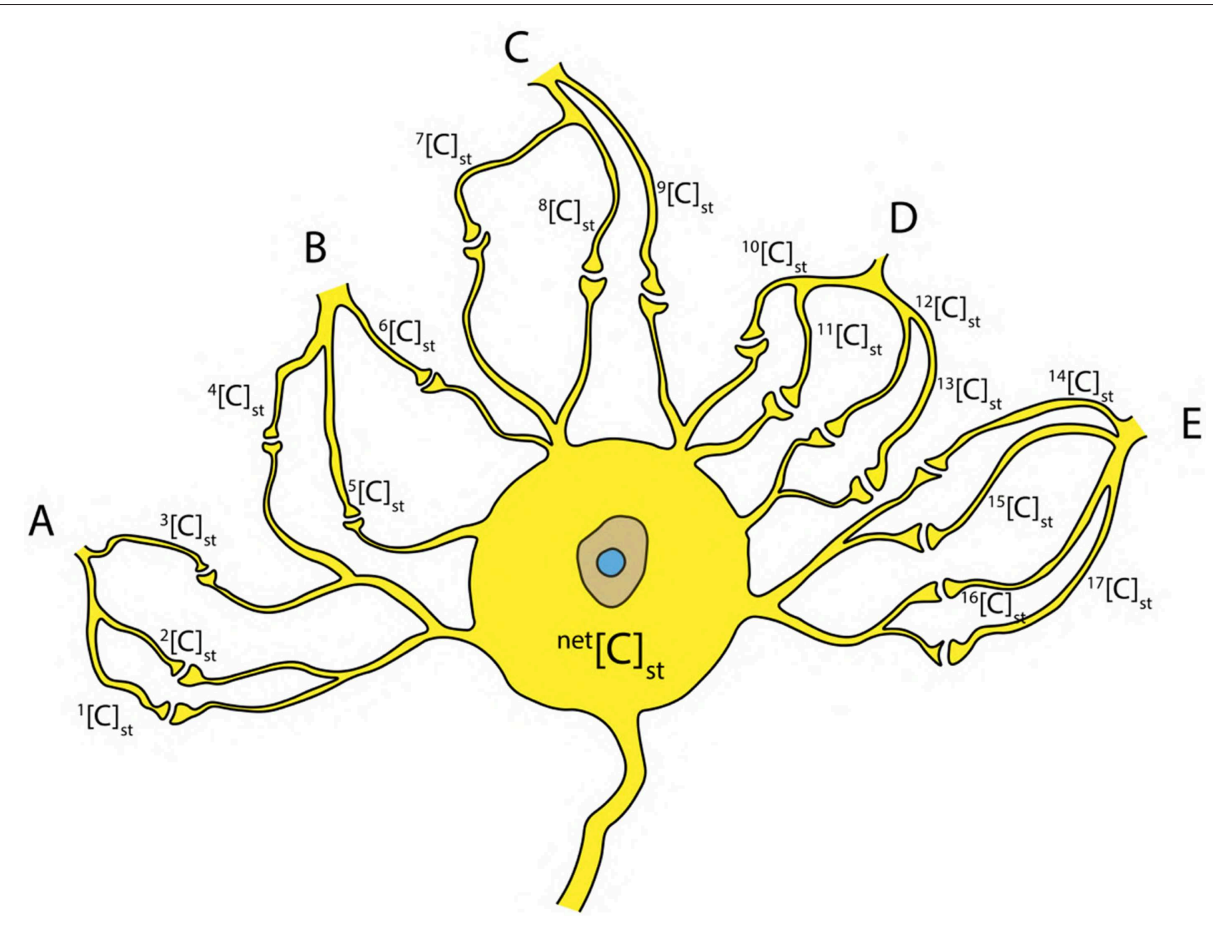

FIGURE 10 | Inputs from neighboring neurons (A-E) to the cell body resulting in a net concentration due to stimuli ${ }^{\text {net }}[\mathrm{C}]_{\mathrm{st}}$ at the axon hillock.

can be generalized for $k$ inputs (as in Equation 13). Hence, the concentration ${ }^{\text {net }}[\mathrm{C}]_{\text {st }}$ can be written as a linear combination (using Equation 11) of critical concentration $[\mathrm{C}]_{\text {crit }}$ (as in Equation 14) such that, it has one to one correspondence with the terms in Equation (13).

${ }^{\text {net }}[\mathrm{C}]_{\mathrm{st}}={ }^{1}[\mathrm{C}]_{\mathrm{st}}+{ }^{2}[\mathrm{C}]_{\mathrm{st}}+\ldots+{ }^{17}[\mathrm{C}]_{\mathrm{st}}$

${ }^{n e t}[\mathrm{C}]_{\mathrm{st}}={ }^{1}[\mathrm{C}]_{\mathrm{st}}+{ }^{2}[\mathrm{C}]_{\mathrm{st}}+\ldots+{ }^{\mathrm{k}}[\mathrm{C}]_{\mathrm{st}}$

${ }^{\text {net }}[\mathrm{C}]_{\mathrm{st}}=\beta_{1}[\mathrm{C}]_{\text {crit }}+\beta_{2}[\mathrm{C}]_{\text {crit }}+\ldots+\beta_{\mathrm{k}}[\mathrm{C}]_{\text {crit }}=\sum_{\mathrm{i}=1}^{\mathrm{k}} \beta_{\mathrm{i}}[\mathrm{C}]_{\text {crit }}$

Multiplication factor $\beta$ (in Equation 11) is decided by the ratio of, net concentration ${ }^{n e t}[\mathrm{C}]_{\text {st }}$ and the critical concentration $[\mathrm{C}]_{\text {crit }}$ as in Equation 15. This factor decides the number of AP (or spike rate of $\mathrm{AP}$ ) for a given stimuli which is nothing but, the frequency $\mathrm{f}_{\text {ribbon }}$ (as in Equation 16). It can be deduced that, the concentration ${ }^{\text {net }}[\mathrm{C}]_{\text {st }}$ is a factor which decides the frequency of oscillations $\mathrm{f}_{\text {ribbon }}$ (i.e., spike rate of AP). This is justified by an illustration, consider a stimuli which acts on a neuron for time (t) such that, the concentration ${ }^{n e t}[\mathrm{C}]_{s t}$ is $\mathbf{1 0 0} \mathrm{mmol} / \mathrm{L}$, then, for a critical concentration (required to generate an AP) say $[\mathrm{C}]_{\text {crit }}=\mathbf{2 0} \mathrm{mmol} / \mathrm{L}$, the multiplication factor $\beta$ turns out to be 5. Therefore, according to Equation (16), the frequency $f_{\text {ribbon }}$ should be $\simeq 5$ per unit time $(t)$. It turns out that, the frequency $f_{\text {ribbon }}$ is governed by RF (which depends on the functions of $\mathrm{Na}^{+}-\mathrm{K}^{+}$pump, $\mathrm{Na}^{+}, \mathrm{K}^{+}$ion leak channels, Figure 1A) and can vary with respect to constant of elasticity (k) of the ribbon [i.e., elasticity of the nerve membrane discussed in Heimburg (2009)].

$$
\begin{array}{r}
\beta=\frac{{ }^{\text {net }}[\mathrm{C}]_{\mathrm{st}}}{[\mathrm{C}]_{\text {crit }}} \\
\beta \propto \mathrm{f}_{\text {ribbon }}
\end{array}
$$

The periodic time (Halliday et al., 1995) of oscillations (T) is a function of RF i.e., $T_{R F}$. It is related to the constant of elasticity $(\mathrm{k})$, net mass $(\mathrm{m})$ of a section and the frequency of ribbon oscillations $f_{\text {ribbon }}$ as in Equations (17) and (18), respectively.

$$
\begin{aligned}
\mathrm{T}_{\mathrm{RF}} & =2 \pi \sqrt{\frac{\mathrm{m}}{\mathrm{k}}} \\
\mathrm{f}_{\text {ribbon }} & =\frac{1}{\mathrm{~T}_{\mathrm{RF}}}
\end{aligned}
$$

Then, the frequency $f_{\text {ribbon }}$ is related to the net strength of the stimuli ${ }^{\text {net }} E_{\text {st }}$ as in Equation (19) (obtained using Equations 10, 15 , and 16).

$$
\mathrm{f}_{\text {spike }} \sim \mathrm{f}_{\text {ribbon }}=\frac{1}{2 \pi} \sqrt{\frac{\mathrm{k}}{\mathrm{m}}} \propto \frac{{ }_{\text {net }}[\mathrm{C}]_{\text {st }}}{[\mathrm{C}]_{\text {crit }}}
$$

Hence, for a unit length of the nerve membrane which is approximated as an elastic ribbon and SM system (Figure 5), the frequency $f_{\text {ribbon }}$ has a dependence on the strength of stimuli (in terms of ion concentrations [C]) as in Equation (19). This frequency is the spike rate of AP represented by $\mathrm{f}_{\text {spike }}$. 


\section{DISCUSSION}

The major question persistent in the field of neurobiology is, how do neurons encode information and transmit it across the axon as an AP? How is the strength of stimuli correlated to the spike rate of AP? What is the physiological role of the nerve membrane in this regard?

Although, the HH model (Hodgkin and Katz, 1949; Hodgkin and Huxley, 1952a,b; Hodgkin, 1964a,b) treats nerve membrane in a purely electric regime, it does not justify the key physiological features which is the reason for its criticism (Drukarch et al., 2018). But, the correlation between strength of stimuli and the spike rate of AP (which is how cells encode information) in terms of variation in the input/stimuli current can be easily established from the $\mathrm{HH}$ model. Prior to the pioneering work by $\mathrm{HH}$, Wilke (1912) had proposed a Pressure Wave theory for AP, indicating toward the physiological changes in nerve membrane successive to an AP event but, missed out on correlating the strength of stimuli and the spike rate of AP. Also, the theory proposed by Wilke did not gain much of attention until, the advent of sophisticated experimental techniques which proved that, the AP is accompanied with certain physiological changes (Hill, 1950; Abbott et al., 1958; Julian and Goldman, 1962; Biondi et al., 1972; Hill et al., 1977; Iwasa and Tasaki, 1980; Iwasa et al., 1980; Tasaki and Iwasa, 1982; Terakawa, 1985; Tasaki et al., 1989; Tasaki and Byrne, 1992a,b; Galbraith et al., 1993; Tasaki, 1999). These interpretations are polarized into two school of thoughts, one which revolved around the electrical interpretation and the other around non-electrical interpretation. This bifurcation continued, until recently when, new theories were proposed to address the empirical observations by combining the electrical features with non-electrical (mechanical/thermodynamic) features of the nerve membrane pertaining to AP. ST by Heimburg-Jackson (Heimburg and Jackson, 2005, 2007), Rvachev model (Rvachev, 2010), and MSW interpretation by Hady-Machta (El Hady and Machta, 2015) have resulted in successful incorporation of the physiological changes accompanying AP. These models present a mixed interpretation of nerve membrane i.e., ST model (Heimburg and Jackson, 2005, 2007) addresses mechanical and thermodynamic nature of nerve membrane whereas, Rvachev and MSW model indicate toward the interplay of electrical

\section{REFERENCES}

Abbott, B., Hill, A. V., and Howarth, J. V. (1958). The positive and negative heat production associated with a nerve impulse. Proc. R. Soc. Lond. B 148, 149-187. doi: $10.1098 / \mathrm{rspb} .1958 .0012$

Adrian, E. D. (1914). The all-or-none principle in nerve. J. Physiol. 47, 460-474. doi: 10.1113/jphysiol.1914.sp001637

Andersen, S. S., Jackson, A. D., and Heimburg, T. (2009). Towards a thermodynamic theory of nerve pulse propagation. Prog. Neurobiol. 88, 104113. doi: $10.1016 /$ j.pneurobio.2009.03.002

Bean, B. P. (2007). The action potential in mammalian central neurons. Nat. Rev. Neurosci. 8:451. doi: 10.1038/nrn2148

Bellhouse, B. (1972). Fluid mechanics of a model mitral valve and left ventricle. Cardiovasc. Res. 6, 199-210. doi: 10.1093/cvr/6.2.199

Bellhouse, B. J., and Bellhouse, F. H. (1969). Fluid mechanics of the mitral valve. Nature 224:615. doi: 10.1038/224615a0

Biondi, R. J., Levy, M. J., and Weiss, P. A. (1972). An engineering study of the peristaltic drive of axonal flow. Proc. Natl. Acad. Sci. U.S.A. 69, 1732-1736. doi: $10.1073 /$ pnas.69.7.1732 and mechanical nature of nerve membrane. This "hit and miss" scenario necessitates the development of a model which can unify them and establish a correlation between the strength of stimuli and spike rate (frequency) of AP (which is purely electrical and fundamental to every neural activity).

A novel approach is proposed in this paper to unify the well established theories encompassing delicate and important features of nerve membrane from a thorough biophysical and physiological perspective. From the VT it is suggested that, the vortex ring formation leads to variation in intracellular and extracellular pressure indicating that, the motion of AP through the axon of a neuron is peristaltic in nature which, justifies the increase and decrease in axon diameter, pressure and length observed empirically. The experimental verification of vortex ring formation is possible by performing high speed patch-clamp fluorometry (generally used to study ion channel conformations Bullen et al., 1997; Harms et al., 2003; Kusch and Zifarelli, 2014; Talwar and Lynch, 2015; Wulf and Pless, 2018) with spatio-temporal accuracy using fluorescent indicators for ion concentrations (Tsien, 1989). As far as the absorptionemission heat and variation in optical properties is concerned, the interpretation from ST, Rvachev and MSW model is retained. The MOT describes, membrane oscillations driven by the vortex ring formation which is used establish quantitatively, a correlation between the strength of stimuli and the spike rate of AP.

Hence, from a macroscopic (neglecting the complexities of molecular cell biology to some extent) perspective, VT and MOT unifies ST, Rvachev and MSW models. It is concluded that, MOT can be used to develop of novel computational tools to mimic neural activity with better accuracy.

\section{AUTHOR CONTRIBUTIONS}

The corresponding author has individually developed the theory, illustrations and wrote the manuscript.

\section{ACKNOWLEDGMENTS}

Author acknowledges, Dr. Benjamin Drukarch, Prof. Bhagya M Sattigeri, Prof. Prafulla K Jha and Dr. Debjani Bagchi for their encouraging discussions and insightful suggestions.

Budvytyte, R., Gonzalez-Perez, A., Mosgaard, L., and Heimburg, T. (2014). Action potential collision in nerves. Biophys. J. 106:794a. doi: 10.1016/j.bpj.2013.11.4356

Bullen, A., Patel, S. S., and Saggau, P. (1997). High-speed, random-access fluorescence microscopy: I. High-resolution optical recording with voltage-sensitive dyes and ion indicators. Biophys. J. 73, 477-491. doi: 10.1016/S0006-3495(97)78086-X

Catterall, W. A. (1995). Structure and function of voltage-gated ion channels. Annu. Rev. Biochem. 64, 493-531. doi: 10.1146/annurev.bi.64.070195.002425

Catterall, W. A., Raman, I. M., Robinson, H. P., Sejnowski, T. J., and Paulsen, O. (2012). The hodgkin-huxley heritage: from channels to circuits. J. Neurosci. 32, 14064-14073. doi: 10.1523/JNEUROSCI.3403-12.2012

Debanne, D. (2004). Information processing in the axon. Nat. Rev. Neurosci. 5:304. doi: $10.1038 / \mathrm{nrn} 1397$

Debanne, D., Bialowas, A., and Rama, S. (2013). What are the mechanisms for analogue and digital signalling in the brain? Nat. Rev. Neurosci. 14:63. doi: 10.1038/nrn3361

Drukarch, B., Holland, H. A., Velichkov, M., Geurts, J. J. G., Voorn, P., Glas, G., et al. (2018). Thinking about the nerve impulse: a critical analysis of 
the electricity-centered conception of nerve excitability. Prog. Neurobiol. 169, 172-185. doi: 10.1016/j.pneurobio.2018.06.009

El Hady, A., and Machta, B. B. (2015). Mechanical surface waves accompany action potential propagation. Nat. Commun. 6:6697. doi: 10.1038/ncomms7697

Engelbrecht, J., Peets, T., Tamm, K., Laasmaa, M., and Vendelin, M. (2016). On modelling of physical effects accompanying the propagation of action potentials in nerve fibres. arXiv: 1601.01867 .

Fields, R. D. (2011). Signaling by neuronal swelling. Sci. Signal. 4:tr1. doi: $10.1126 /$ scisignal.4155tr1

Fillafer, C., Mussel, M., Muchowski, J., and Schneider, M. F. (2018). Cell surface deformation during an action potential. Biophys. J. 114, 410-418. doi: 10.1016/j.bpj.2017.11.3776

Galbraith, J. A., Thibault, L. E., and Matteson, D. R. (1993). Mechanical and electrical responses of the squid giant axon to simple elongation. J. Biomech. Eng. 115, 13-22. doi: 10.1115/1.2895464

Hall, J. E. (2010). Guyton and Hall Textbook of Medical Physiology e-Book. Amsterdam: Elsevier Health Sciences.

Halliday, D., Resnick, R., and Walker, J. (1995). Fundamentals of Physics, Chapters 1-12, Vol. 1, New Jersey, NJ: Wiley.

Harmon, L., and Lewis, E. R. (1966). Neural modeling. Physiol. Rev. 46, 513-591. doi: 10.1152 /physrev.1966.46.3.513

Harms, G. S., Orr, G., Montal, M., Thrall, B. D., Colson, S. D., and Lu, H. P. (2003). Probing conformational changes of gramicidin ion channels by singlemolecule patch-clamp fluorescence microscopy. Biophys. J. 85, 1826-1838. doi: 10.1016/S0006-3495(03)74611-6

Heimburg, T. (2009). Physical properties of biological membranes. arXiv: 0902.2454.

Heimburg, T., and Jackson, A. D. (2005). On soliton propagation in biomembranes and nerves. Proc. Natl. Acad. Sci. U.S.A. 102, 9790-9795. doi: $10.1073 /$ pnas. 0503823102

Heimburg, T., and Jackson, A. D. (2007). On the action potential as a propagating density pulse and the role of anesthetics. Biophys. Rev. Lett. 2, 57-78, doi: 10.1142/S179304800700043X

Hill, B. C., Schubert, E. D., Nokes, M. A., and Michelson, R. P. (1977). Laser interferometer measurement of changes in crayfish axon diameter concurrent with action potential. Science 196, 426-428. doi: 10.1126/science. 850785

Hill, D. K. (1950). The volume change resulting from stimulation of a giant nerve fibre. J. Physiol. 111, 304-327. doi: 10.1113/jphysiol.1950.sp004481

Hille, B. (2001). Ion Channels of Excitable Membranes, Vol. 507. Sunderland, MA: Sinauer.

Hodgkin, A. L. (1964a). The Conduction of the Nervous Impulse, Liverpool: Liverpool University.

Hodgkin, A. L. (1964b). The ionic basis of nervous conduction. Science 145, 1148-1154. doi: 10.1126/science.145.3637.1148

Hodgkin, A. L., and Huxley, A. F. (1952a). Currents carried by sodium and potassium ions through the membrane of the giant axon of loligo. J. Physiol. 116, 449-472. doi: 10.1113/jphysiol.1952.sp004717

Hodgkin, A. L., and Huxley, A. F. (1952b). A quantitative description of membrane current and its application to conduction and excitation in nerve. J. Physiol. 117, 500-544.

Hodgkin, A. L., and Katz, B. (1949). The effect of sodium ions on the electrical activity of the giant axon of the squid. J. Physiol. 108, 37-77. doi: 10.1113/jphysiol.1949.sp004310

Hooke, R. (1635-1703). Lectures de Potentia Restitutiva, or, Of Spring, Explaining the Power of Springing Bodies. London: Printed for John Martyn printer to the Royal Society.

Iwasa, K., and Tasaki, I. (1980). Mechanical changes in squid giant axons associated with production of action potentials. Biochem. Biophys. Res. Commun. 95, 1328-1331. doi: 10.1016/0006-291x(80)91619-8

Iwasa, K., Tasaki, I., and Gibbons, R. C. (1980). Swelling of nerve fibers associated with action potentials. Science 210, 338-339. doi: 10.1126/science.7423196

Julian, F. J., and Goldman, D. E. (1962). The effects of mechanical stimulation on some electrical properties of axons. J. Gen. Physiol. 46, 297-313. doi: 10.1085/jgp.46.2.297

Kim, G. H., Kosterin, P., Obaid, A. L., and Salzberg, B. M. (2007). A mechanical spike accompanies the action potential in mammalian nerve terminals. Biophys. J. 92, 3122-3129. doi: 10.1529/biophysj.106.103754

Kusch, J., and Zifarelli, G. (2014). Patch-clamp fluorometry: electrophysiology meets fluorescence. Biophys. J. 106, 1250-1257. doi: 10.1016/j.bpj.2014. 02.006
Lodish, H., Berk, A., Zipursky, S. L., Matsudaira, P., Baltimore, D., Darnell, J., et al. (1995). Molecular Cell Biology, Vol. 3. New York, NY: WH Freeman.

Mueller, J. K., and Tyler, W. J. (2014). A quantitative overview of biophysical forces impinging on neural function. Phys. Biol. 11:051001. doi: 10.1088/1478-3975/11/5/051001

Pasipoularides, A., Murgo, J. P., Bird, J. J., and Craig, W. E. (1984). Fluid dynamics of aortic stenosis: mechanisms for the presence of subvalvular pressure gradients. Am. J. Physiol. Heart Circul. Physiol. 246, H542-H550. doi: 10.1152/ajpheart.1984.246.4.H542

Purves, D., Cabeza, R., Huettel, S. A., LaBar, K. S., Platt, M. L., Woldorff, M. G., et al. (2008). Cognitive Neuroscience. Sunderland, MA: Sinauer Associates, Inc.

Reul, H., Talukder, N., and Müller, E. W. (1981). Fluid mechanics of the natural mitral valve. J. Biomech. 14, 361-372. doi: 10.1016/0021-9290(81)90046-4

Rvachev, M. M. (2010). On axoplasmic pressure waves and their possible role in nerve impulse propagation. Biophys. Rev. Lett. 5, 73-88. doi: $10.1142 / S 1793048010001147$

Safran, S. (2018). Statistical Thermodynamics Of Surfaces, Interfaces, And Membranes. Florida, FL: CRC Press.

Sedov, L. I. (1997). Mechanics of Continuous Media (IN 2 VOLS) (Vol. 4). Singapore: World Scientific Publishing Company.

Seo, J. H., Vedula, V., Abraham, T., Lardo, A. C., Dawoud, F., Luo, H., et al. (2014). Effect of the mitral valve on diastolic flow patterns. Phys. Fluids 26:121901. doi: $10.1063 / 1.4904094$

Shariff, K., and Leonard, A. (1992). Vortex rings. Annu. Rev. Fluid Mech. 24, 235-279. doi: 10.1146/annurev.fl.24.010192.001315

Shrivastava, S. (2014). Non-linear solitary sound waves in lipid membranes and their possible role in biological signaling. (Ph.D. thesis). Boston University, Boston, MA, United States.

Talwar, S., and Lynch, J. W. (2015). Investigating ion channel conformational changes using voltage clamp fluorometry. Neuropharmacology 98, 3-12. doi: 10.1016/j.neuropharm.2015.03.018

Tasaki, I. (1999). Rapid structural changes in nerve fibers and cells associated with their excitation processes. Japanese J. Physiol. 49, 125-138. doi: 10.2170/jjphysiol.49.125

Tasaki, I., and Byrne, P. (1992a). Rapid structural chances in nerve fibers evoked by electric current pulses. Biochem. Biophys. Res. Commun. 188, 559-564. doi: 10.1016/0006-291x(92)91092-5

Tasaki, I., and Byrne, P. M. (1992b). Heat production associated with a propagated impulse in bullfrog myelinated nerve fibers. Japanese J. Physiol. 42, 805-813. doi: $10.2170 /$ jjphysiol.42.805

Tasaki, I., and Iwasa, K. (1982). Rapid pressure changes and surface displacements in the squid giant axon associated with production of action potentials. Japanese J. Physiol. 32, 69-81. doi: 10.2170/jiphysiol.32.69

Tasaki, I., Kusano, K., and Byrne, P. (1989). Rapid mechanical and thermal changes in the garfish olfactory nerve associated with a propagated impulse. Biophys. J. 55, 1033-1040. doi: 10.1016/S0006-3495(89)82902-9

Terakawa, S. (1985). Potential-dependent variations of the intracellular pressure in the intracellularly perfused squid giant axon. J. Physiol. 369, 229-248. doi: 10.1113 /jphysiol.1985.sp015898

Tsien, R. Y. (1989). "Fluorescent indicators of ion concentrations," in Methods in Cell Biology, Vol. 30, eds D. L. Taylor and Y.-L. Wang (Cambridge, MA: Academic Press), 127-156.

Tyler, W. J. (2012). The mechanobiology of brain function. Nat. Rev. Neurosci. 13:867. doi: $10.1038 / \mathrm{nrn} 3383$

Wilke, E. (1912). Das problem der reizleitung im nerven vom standpunkte der wellenlehre aus betrachtet. Pflüger's Archiv für die gesamte Physiologie des Menschen und der Tiere 144, 35-38. doi: 10.1007/BF01681175

Wulf, M., and Pless, S. A. (2018). High-sensitivity fluorometry to resolve ion channel conformational dynamics. Cell Rep. 22, 1615-1626. doi: 10.1016/j.celrep.2018.01.029

Conflict of Interest: The author declares that the research was conducted in the absence of any commercial or financial relationships that could be construed as a potential conflict of interest.

Copyright (C) 2020 Sattigeri. This is an open-access article distributed under the terms of the Creative Commons Attribution License (CC BY). The use, distribution or reproduction in other forums is permitted, provided the original author(s) and the copyright owner(s) are credited and that the original publication in this journal is cited, in accordance with accepted academic practice. No use, distribution or reproduction is permitted which does not comply with these terms. 